\title{
Label-free Biosensors on Silicon-on-Insulator Optical Chips
}

\author{
Katrien De $\operatorname{Vos}^{a}$, Peter Debackere ${ }^{a}$, Tom Claes ${ }^{a}$, Jordi Girones ${ }^{b}$, Wout De Cort $^{a}$, Etienne \\ Schacht $^{b}$, Roel Baets ${ }^{a}$ and Peter Bienstman ${ }^{a}$ \\ ${ }^{a}$ Ghent University - Imec \\ Department of Information Technology \\ Sint-Pietersnieuwstraat 41 \\ 9000, Gent, Belgium \\ ${ }^{b}$ Ghent University \\ Polymer Material Research Group \\ Krijgslaan 281 \\ 9000, Gent, Belgium
}

\begin{abstract}
To come up to the demand for extremely sensitive biosensors for parallel real-time bioanalyses, we present several configurations of label-free biosensors on Silicon-on-Insulator (SOI) optical chips. We discuss results on microring resonators with a non-fouling polymer coating, increased sensitivity with slotted wire resonators and the design and fabrication of an integrated surface plasmon resonance interferometer. The high refractive index contrast of SOI offers submicron-size features with high quality for dense integration, high sensitivity and detection with very low analyte volumes. The fabrication method, 193nm deep-UV lithography, allows for mass production of cheap disposable biochips.
\end{abstract}

Keywords: silicon-on-insulator, biosensor, integrated optics, microring resonator, slotted waveguides, plasmonics, interferometer

\section{INTRODUCTION}

While labeled detection methods can be sensitive down to a single molecule, labels can structurally and functionally alter the assay and the labeling process is labor intensive and costly. Quantification is difficult since the bias label intensity level is dependent on all working conditions. Direct biosensors overcome specificity, reliability and durability problems induced by labels. Detection is done in real-time, which is important for kinetic interaction studies and point-of-care diagnostics. Next generation biosensors need to be sensitive, reliable and portable. Silicon-on-Insulator (SOI) allows the reuse of standard high quality CMOS processing facilities, in particular 193 $\mathrm{nm}$ lithography, for fabrication of photonics micro- and nanodevices. ${ }^{1,2}$ This opens the route for very cheap, potentially disposable devices. Thanks to the high index contrast, SOI is suitable for the fabrication of micronand submicron sized optical cavities of very high quality. The combination of low cost fabrication and high sensitivity through small dimensions has proven SOI a good candidate for disposable biosensor array chips.

\section{MICRORING RESONATORS FOR SELECTIVE BIOSENSING}

Label-free biosensing with optical microcavities is considered to be a very promising technique due to its high sensitivity and its potential for integration in multidimensional arrays. ${ }^{3-5}$ A binding event in the near vicinity of an optical cavity will change the local refractive index, which results in a change of the effective refractive index of the optical mode and hence in a resonance wavelength shift. Unlike the sensitivity of interferometers, the sensitivity of optical cavities does not decrease with decreasing sensing area.

Send correspondence to Katrien De Vos,

E-mail: Katrien.Devos@intec.UGent.be 


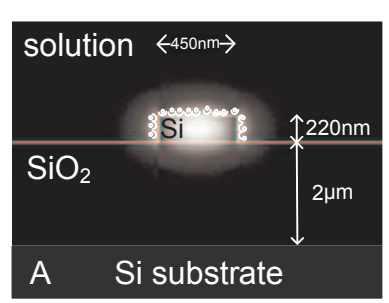

$\mathrm{B}$
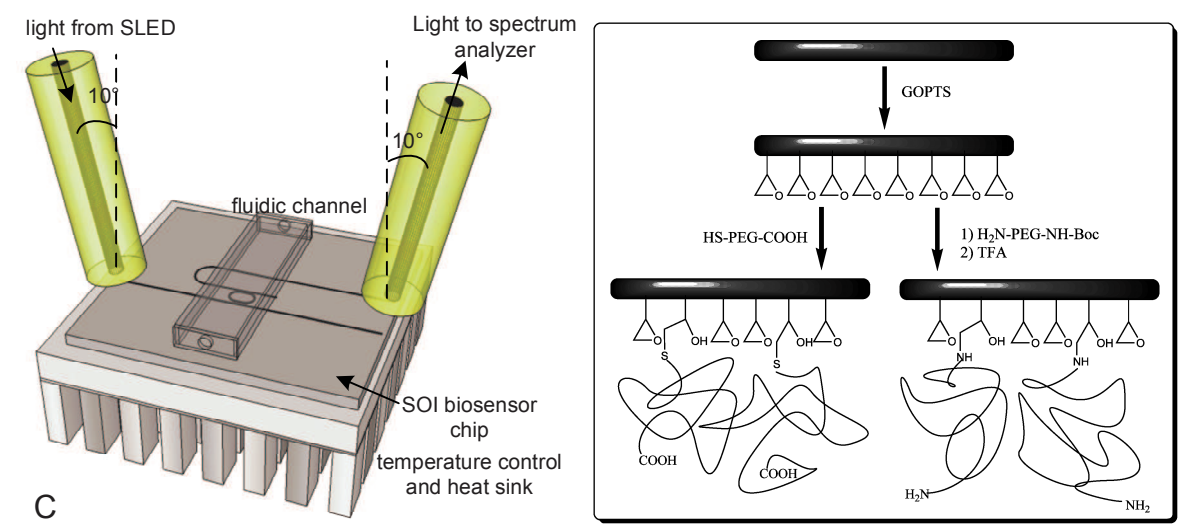

Figure 1: Left: (A) SOI waveguide for evanescent field biosensing (not to scale), (B) SEM picture of an SOI racetrack resonator with radius $5 \mu \mathrm{m}$ and straight sections $20 \mu \mathrm{m}$, (C)Illustration of measurement setup. Right: Modification of silicon surface with PEG layer.

The microring resonators for these experiments are racetracks with $5 \mu m$ radius and $2 \mu m$ straight section for good coupling control. Waveguide dimensions are 220x450nm (figure 1(left) A and B). The resonators have a Q-factor of up to 20,000 with finesse of 240. A high internal Q-factor corresponds to small peaks and hence to a high detection resolution. Moreover the higher the Q-factor, the greater the fitting accuracy. However, high Q-factors set high demands to the equipment resolution and the Q-factor is related to the physical size of the resonator. The smaller the ring, the smaller the amount of molecules needed for full coverage. A Q-factor of 20,000 holds a good compromise between small surface area, small peak width and maximal extinction.

Light is coupled from single mode fibers at $10^{\circ}$ off normal to the waveguides on the SOI chip through grating couplers. ${ }^{1}$ All measurements in 2.2 are performed with a superluminescent LED and an optical spectrum analyser. Spectra are stored every 3 seconds. After fitting all data to a Lorentzian, the resonance wavelength is plotted versus time. A flow cell is mounted on top of the chip and connected through tubings to a Harvard syringe pump (figure 1 (left) C).

\subsection{Poly(ethylene glycol) layer for highly selective biosensing}

The properties of a biosensor critically depend on the quality of the interfacial layer, especially for detection in complex samples. The interfacial layer has to allow immobilization of receptor molecules and at the same time effectively block non-specific interactions with the macromolecular components of the analyzed sample. Homogeneous, thin layers are required that are stable, do not affect the sensors sensitivity and do not hinder transport of compounds to the surface. The coatings of low molecular silanes typically do not have sufficient resistance to non-specific adsorption. We improved this by attaching an ultra thin layer of hydrophilic poly(ethylene glycol) (PEG) in a two step process (figure 1 (right)). The PEG layer is coupled to a silane layer bearing epoxide groups. ${ }^{6}$ We examined two heterobifunctional PEG's: $\alpha$-sulfanyl- $\omega$-carboxy PEG (HS-PEG-COOH) and monoprotected diamino-PEG (H2N-PEG-NH-Boc). The HS-PEG-COOH and H2N-PEG-NH-Boc layers have a thickness of respectively 2.3 and $2.5 \mathrm{~nm}$ and have a surface loading of respectively $99 \mathrm{pm} / \mathrm{cm}^{2}$ carboxygroups and $97 \mathrm{pm} / \mathrm{cm}^{2}$ aminogroups. The contact angles were reproducible $\left(32^{\circ} \pm 1^{\circ}\right)$, lower than the GOPTS-surface contact angle and comparable to literature values.

\subsection{Selective biosensing}

The high affinity avidin/biotin couple has been used as a model biosensing couple to demonstrate repeatability and detection capabilities of the microring resonators. Bovine Serum Albumin (BSA), a protein with similar molecular weight to avidin but with low affinity to biotin, has been used as a model for non-specific interactions. After deprotection of the Boc groups, chips coated with H2N-PEG-NH-Boc were biotinylated. Figure 2 (left) shows the response signal of the chips to a range of avidin concentrations; $2,10,87.5$ and $175 \mu \mathrm{g} / \mathrm{ml}$ and two response signals of the chips to BSA solution at concentration $1 \mathrm{mg} / \mathrm{ml}$. Every curve corresponds to a different experiment on a different chip. For high concentrations, a fast wavelength shift is initially recorded due to the 

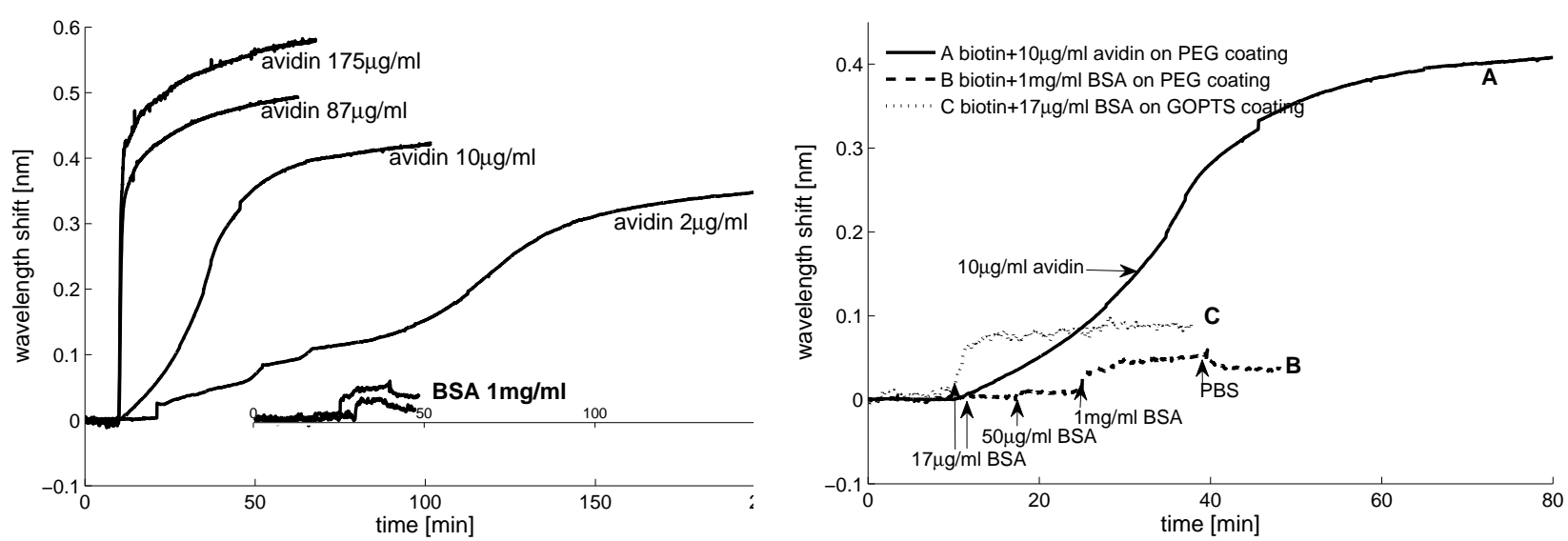

Figure 2: Left: Specific versus non-specific binding tests. Right: Comparison of chips with and without PEG coating.

sudden concentration gradient. Then, the response increases more gradually due to the slow transport of the molecules to the surface. From the saturation levels we can extrapolate a sensitivity of $10 \mathrm{ng} / \mathrm{ml}$ corresponding to the noise level of the fitted data $(2 \mathrm{pm})$, which is in the same range as the sensitivity reported for the 3aminopropyltriethoxy silane (APTES) coated microring resonators. ${ }^{7}$ This compares well with commercially available label-free protein detection methods. Figure 2 (right) shows a comparison of the signal provided by chips with and without the PEG coating. Curve A is the interaction of $10 \mu \mathrm{g} / \mathrm{ml}$ avidin with a biotinylated PEG coated chip. The response signal to avidin arises 210 times above the noise level. Curve $\mathrm{C}$ shows the interaction of $17 \mu \mathrm{g} / \mathrm{ml}$ BSA with a chip without PEG coating. It is clear that even for this small BSA concentration, in absence of the PEG coating, a rather high output is measured. Curve B shows the signal obtained by applying increasing BSA concentrations on a PEG coated chip. For a BSA concentration of $50 \mu \mathrm{g} / \mathrm{ml}$ the signal exceeded only 2.5 times the noise level. For a $1 \mathrm{mg} / \mathrm{ml} \mathrm{BSA} \mathrm{concentration} \mathrm{the} \mathrm{response} \mathrm{was} \mathrm{only} \mathrm{about} 15$ times the noise level.

Using the vectorial mode solver software Fimmwave, we can simulate the wavelength shift for a layer surrounding the waveguide. For a wavelength shift of $2 p m$, equivalent to the noise level of the fitted data, the minimal detectable thickness can be extracted from the simulated data. Using an approximated constant of $1.33 \mathrm{~g} / \mathrm{cm}^{3}$ as molecular layer mass density and a refractive index of 1.45 , from the minimal detectable thickness of $12 \mathrm{fm}$ we can estimate a minimal detectable mass coverage of about $17 \mathrm{pg} / \mathrm{mm}^{2}$. As mentioned, one of the benefits of using the resonating structures rests on its small size and the fact that their response is not influenced by the sensing area. Thus, with a surface area of only $21.84 \mu \mathrm{m}^{2}$ a minimal mass of $0.37 \mathrm{fg}$ could theoretically be detected.

\section{SLOTTED RACETRACKS}

\subsection{Towards a higher accuracy}

An important route towards an improvement of the sensitivity, and possibly of the accuracy and the detection limit, of this sensor, is to increase the interaction between light in the resonator waveguide and biomolecules attaching to the waveguide surface. When using a normal photonic wire, only the evanescent tail of the wire mode will interact with biomolecules (Figure 3, left). By etching a narrow slot in the middle of the waveguide, a vast fraction of the quasi-TE mode will be concentrated in that slot, ${ }^{8}$ so that more light is concentrated along the bio-activated surface of the slot waveguide (Figure 3, right). This will cause attached molecules to have a larger impact on the propagating light, so that the resonance wavelength shift of a racetrack resonator consisting of slot waveguides will be larger than that of a normal racetrack resonator. This concept already proved to be promising for biosensing. ${ }^{9}$

\subsection{Waveguide design}

Using an eigenmode expansion tool, the slot width S and strip width $\mathrm{W}$ of a silicon slot waveguide were optimized for biosensing. The height of the waveguide was kept constant at $220 \mathrm{~nm}$, as this was already fixed by our 


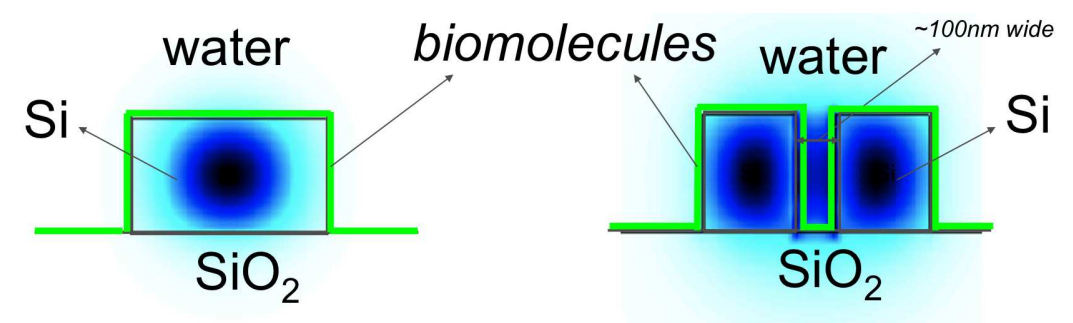

Figure 3: Comparison between the intensity profiles of the quasi-TE modes of a typical silicon photonic wire $(450 \mathrm{~nm}$ wide) and of a silicon slot waveguide $(520 \mathrm{~nm}$ wide with $100 \mathrm{~nm}$ slot width). Both waveguides are $220 \mathrm{~nm}$ high.
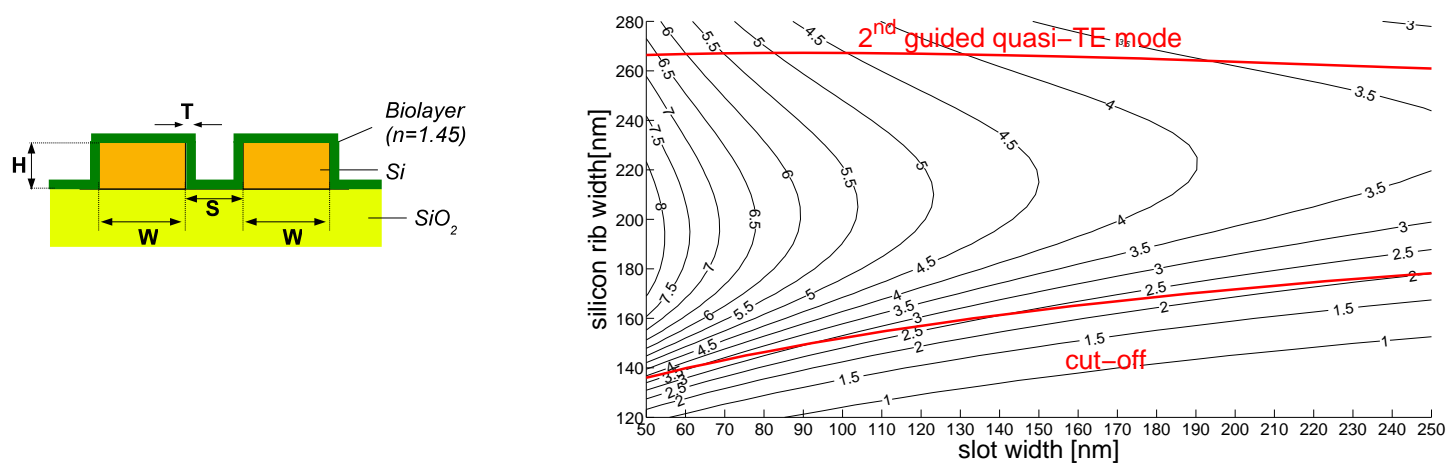

Figure 4: Left: Simulated cross section of the racetrack slot waveguide. A biolayer is used to model the surface chemistry and attaching of biomolecules to the waveguide surface. Right: Simulated shift of the slotted racetrack resonator as a function of the slot width and rib width of the waveguide, when the sensor is saturated with biomolecules (full coverage of the waveguides). The single mode regime for the quasi-TE polarization is indicated.

fabrication process. Surface sensing was simulated by a layer with refractive index 1.45 covering the whole waveguide (Figure 4, left).

As a function of slot width $\mathrm{S}$ and strip width $\mathrm{W}$, the effective index of the waveguide was simulated once for $1 \mathrm{~nm}$ biolayer thickness (model for initial surface chemistry) and once for $6 \mathrm{~nm}$ biolayer thickness (model for saturated surface). Taking first order dispersion into account, the difference in effective index was used to calculated the expected resonance wavelength shift of the racetrack resonator applying this formula:

$$
\Delta \lambda=\frac{\left(n_{e f f, 6 n m}-n_{e f f, 1 n m}\right) \lambda_{r e s}}{n_{g}}
$$

Here, $\lambda_{\text {res }}$ is the initial resonance wavelength of the racetrack resonator and $n_{g}$ is the group index of the slot waveguide at the resonance wavelength.

The graph in Figure 4 shows the calculated resonance wavelength shift as a function of $\mathrm{S}$ and $\mathrm{W}$. In this range of the paramaters, the smaller the slot width $\mathrm{S}$ becomes, the higher the sensitivity will be, and for each value of $\mathrm{S}$, the optimal value for $\mathrm{W}$ can be read from the graph.

\subsection{Fabrication}

Slotted racetrack resonators with $5 \mu m$ radius and $3 \mu m$ straight coupling sections were fabricated with deep-UV lithography. ${ }^{10}$ Figure 5 shows scanning electron microscope images of our slotted racetrack resonator with 100nm slot width. 

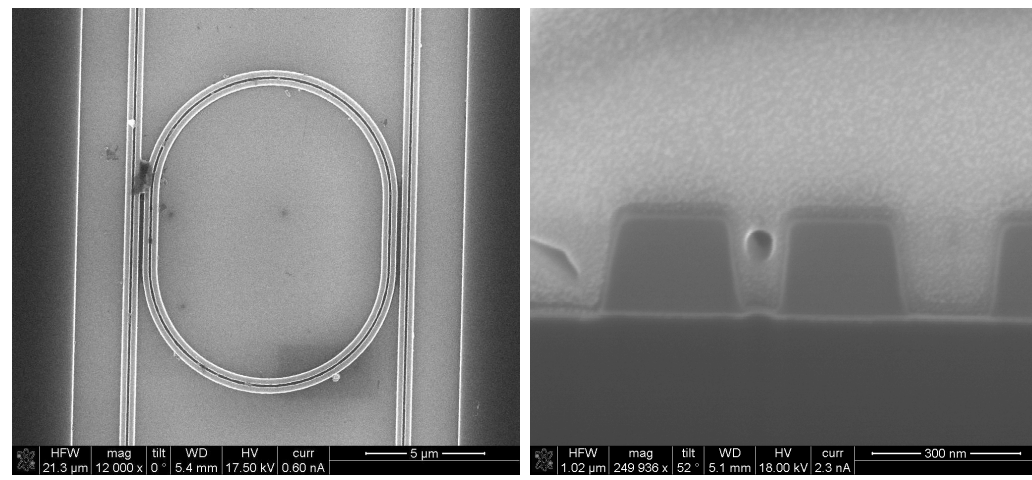

Figure 5: Left: scanning electron microscope image of the slotted racetrack resonator with $5 \mu \mathrm{m}$ radius, fabricated with deep-UV lithography. Right: cross section of the silicon slot waveguide with $100 \mathrm{~nm}$ slot width (measured at half height).

\subsection{Bulk refractive index sensing}

The bulk sensitivity of this slotted racetrack resonator was measured by flowing watery NaCl-solutions with different concentrations over the sensor. A flow cell with closed channel was used to avoid evaporation and no surface chemistry was applied to the sensor surface for this experiment. The left graph in Figure 6 shows the shift of a resonance dip in the pass spectrum of the slotted racetrack, when different salt concentrations are flown over. Lorentzian fitting was used to determine the resonance wavelength. This resonator has a quality factor of 420 .
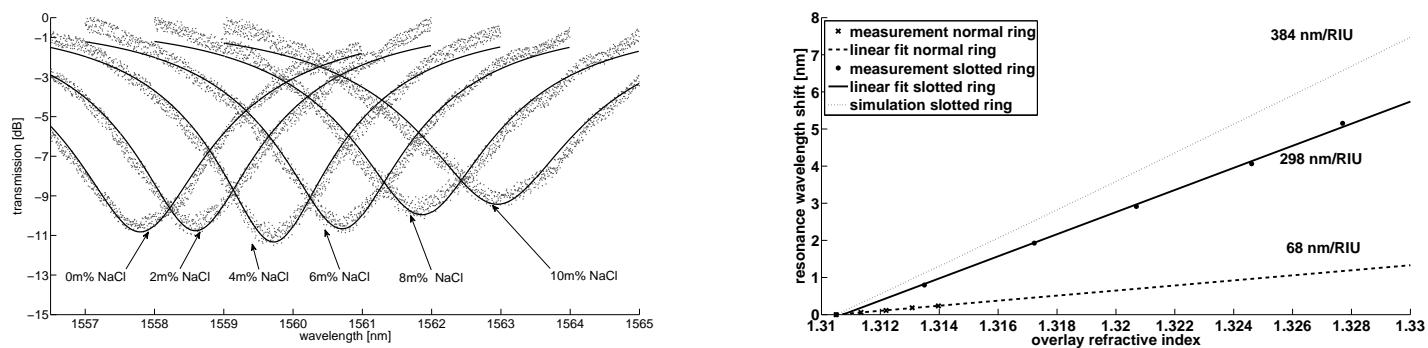

Figure 6: Left: Resonance dip in the pass spectrum of the slotted racetrack resonator for different salt concentrations. Right: Comparison between the experimental resonance wavelength shift of a normal racetrack and the theoretical and experimental resonance wavelength shift of a slotted racetrack as a function of the overlay refractive index. Experimental data ${ }^{11}$ was used to determine the refractive index of the salt concentrations.

The right graph in Figure 6 shows the linear shift of the resonance wavelength as a function of the top cladding index, where the refractive index of the salt solutions was determined ${ }^{11}$ at a wavelength of $1550 \mathrm{~nm}$. The bulk sensitivity of the slotted racetrack resonator with $100 \mathrm{~nm}$ slot region is $298 \mathrm{~nm} / \mathrm{RIU}$ as compared to $68 \mathrm{~nm} / \mathrm{RIU}$ for normal racetracks. Using an eigenmode expansion tool, the effective index change of a slot waveguide with the same dimensions as in Figure 5 was simulated for changing top cladding index. Based on this, a theoretical bulk sensitivity of $384 \mathrm{~nm} / \mathrm{RIU}$ was calculated, taking first order dispersion into account.

\section{SURFACE PLASMON INTERFEROMETER}

Combining Surface Plasmon interrogation techniques and Silicon-on-Insulator (SOI) as a host substrate is another potentially viable route towards lab-on-chip biosensors.

Surface Plasmon Polariton modes are sensitive to refractive index changes due to the very high evanescent fields near the surface on which they propagate, making them suitable for real-time monitoring of solutions and binding events that occur near the sensor surface. ${ }^{12,13}$ Moreover, $A u$ boasts the advantage that it is a 


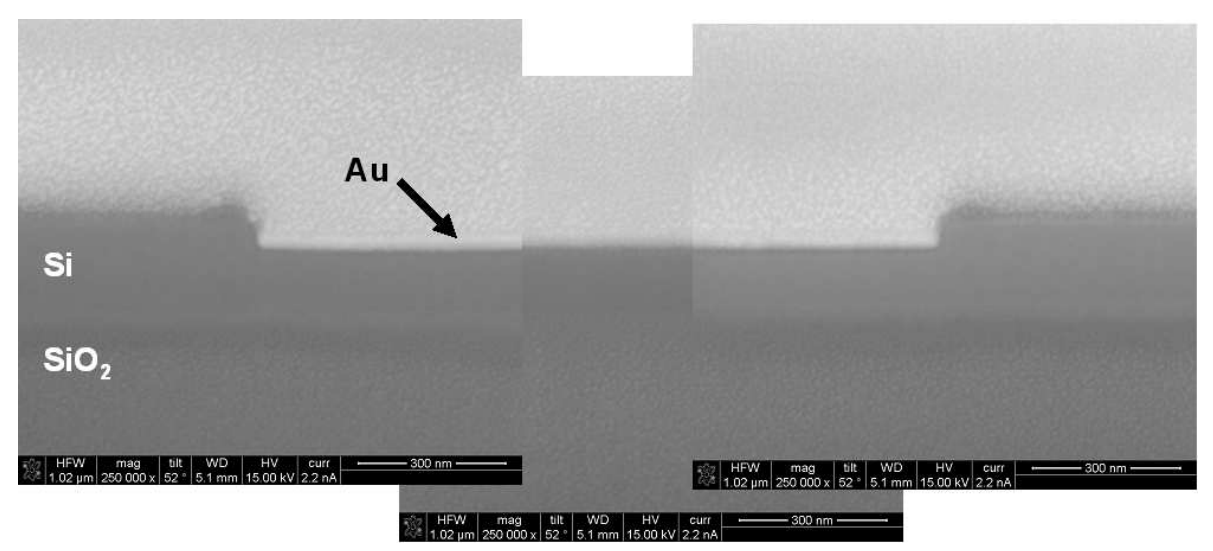

Figure 7: Composite image of three FIB cross-sections of the fabricated samples.

bio-compatible material and that a very mature thiol-chemistry can be used for the functionalisation of this layer. Drawbacks are that state-of-the-art surface plasmon resonance (SPR) devices are bulky, expensive, and the integration with microfluidics and route towards multiparameter analysis are difficult.

Using Silicon-on-Insulator as a host substrate can solve some of these problems plaguing SPR sensors. Waveguide sensors constructed in SOI can have extremely small footprint, can be integrated into compact arrays, and be readily combined with fluidic components, promising features for potential lab-on-a-chip applications. ${ }^{1}$ Furthermore, monolithic integration of sensors with electronics is also feasible.

The principle of a surface plasmon interferometer is based on the interference of two decoupled surface plasmon modes propagating on either side of a thin metallic layer embedded into a silicon waveguide. Decoupling occurs due to the high degree of asymmetry in the refractive index of the cladding layers above and below the $A u$ stripe. The theoretical concept and feasibility were previously reported. ${ }^{14}$ Theoretical results predict that this device would be capable of detecting very small refractive index changes $\left(10^{-6} R I U\right)$ for a device which has a physical footprint of only $100 \mu m^{2}$.

\subsection{Fabrication}

Fabricated and measured samples consist of a silicon substrate, $2 \mu \mathrm{m}$ of high density plasma silicon dioxide and $220 \mathrm{~nm}$ of hydrogenated amorphous silicon $(a-S i: H)$, deposited using a plasma-enhanced chemical vapour deposition process with $\mathrm{SiH}_{4}$ and $\mathrm{He}$ gases, ${ }^{15}$ lithography was done using deep-UV steppers. A protective layer of BCB was used to prevent the flowcell from damaging the $S i$-waveguides. The length of the sensing area was $8 \mu \mathrm{m}$, waveguides were $3 \mu \mathrm{m}$ wide. Etch depth of the sensor region in the $S i$ was $70 \mathrm{~nm}$, the $A u$ layer was deposited using vacuum evaporation and was $37 \mathrm{~nm}$ thick. A focused ion beam cross-section of the fabricated device can be seen in Fig. 7.

\subsection{Measurement results}

Light from a single mode fiber was coupled into the sample using a lensed fiber, outcoupling occurs through an objective. Measurements have been performed using a tunable laser (output power $5 \mathrm{~mW}$ ) and power detector, a polarizer has been used in front of the detector in order to ensure that only TM polarized light is measured.

To quantify the sensitivity of the device with respect to bulk refractive index changes, deionized water, an aqueous solution of $2 m \%, 4 m \%, 6 m \%, 8 m \%$ and $10 m \%$ of $N a C L$ were flown over the sensor surface, using a flow cell to avoid evaporation. The spectra for these measurements are presented in Fig. 8. Standard deviation error bars represent measurements of several spectra with an interval of $10 \mathrm{~min}$ to ensure that the steady-state response was measured. One can clearly see that the position of the spectral minimum is blueshifted as a function of increasing analyte refractive index, which is in good agreement with theoretical predictions. At $20^{\circ} \mathrm{C}$ the refractive index of an aqueous solution of $\mathrm{NaCl}$ varies with $0.0018 \mathrm{RIU}$ (Refractive Index Units) per mass $\%^{16}$. A two-point moving average filter and spectral normalisation has been utilised to analyse he spectral data. Sensitivity $S_{R I U}[n m / R I U]$ for this sensor was determined by calculating the wavelength of destructive 


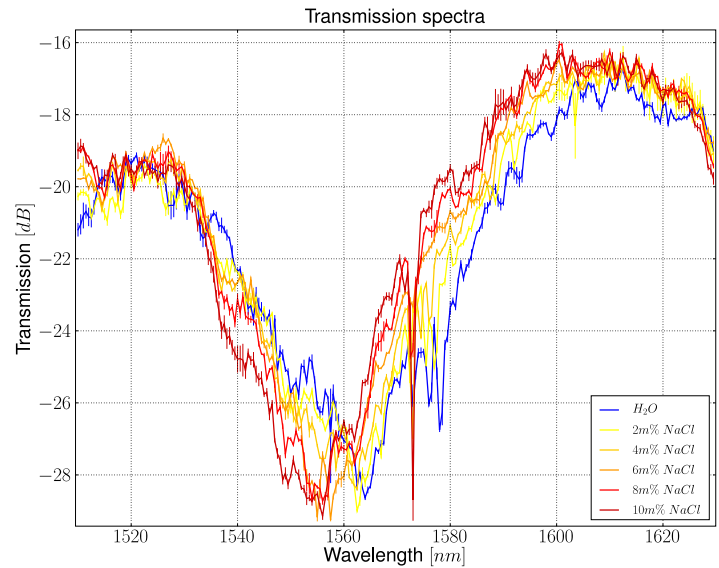

Figure 8: Measured transmission spectra for different NaCl Figure 9: Position of the spectral minimum as a function of concentrations

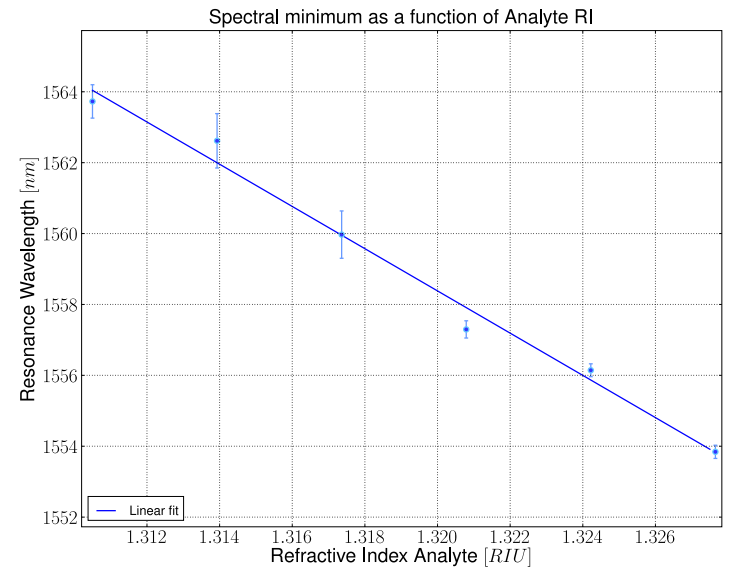

analyte refractive index.

interference using a weighted centroid algorithm, ${ }^{17}$ the standard deviation error $\delta \lambda_{\min }$ is partly due to the dip finding algorithm (dependence of the dip position as a function of treshold value is taken into account) and partly due to measurement errors. Results are given in the table 4.2. Using this data we can estimate the general sensitivity $S_{R I U}$ of this device to be $595.726 \mathrm{~nm} / R I U$. Taking the magnitude of this wavelength shift and the error in determining the exact spectral minimum $\delta \lambda_{\min }$ into account, the detection limit can be calculated, these values are also given in table 4.2. The wavelength of minimal transmission versus the refractive index of the analyte flown over the sensor surface is depicted in Fig. 9.

\begin{tabular}{lcccc} 
Analyte & RI & $\lambda_{\min }[n m]$ & $\delta \lambda_{\min }[n m]$ & $\mathrm{DL}[\mathrm{RIU}]$ \\
\hline $\mathrm{H}_{2} \mathrm{O}$ & 1.3105 & 1563.727 & 0.471 & $7.902 \times 10^{-4}$ \\
$2 \mathrm{~m} \% \mathrm{NaCl}$ & 1.3139 & 1562.617 & 0.767 & $1.288 \times 10^{-3}$ \\
$4 \mathrm{~m} \% \mathrm{NaCl}$ & 1.3173 & 1559.970 & 0.668 & $1.121 \times 10^{-3}$ \\
$6 \mathrm{~m} \% \mathrm{NaCl}$ & 1.3208 & 1557.296 & 0.243 & $4.0815 \times 10^{-4}$ \\
$8 \mathrm{~m} \% \mathrm{NaCl}$ & 1.3242 & 1556.143 & 0.181 & $3.048 \times 10^{-4}$ \\
$10 \mathrm{~m} \% \mathrm{NaCl}$ & 1.3242 & 1553.842 & 0.186 & $3.128 \times 10^{-4}$ \\
\hline \multicolumn{5}{c}{ Sensitivity: $595.726 \pm 0.395 \mathrm{~nm} / \mathrm{RIU}$}
\end{tabular}

\section{CONCLUSION}

We presented several nanophotonic biosensors on silicon-on-insulator: ring resonator based devices, slotted ring resonators to increase the interaction between light and the sample, and finally devices based on nanoplasmonic interferometers. We also presented optimized chemistry to limit non-specific binding.

\section{ACKNOWLEDGMENTS}

This work was funded by Ghent University through the GOA project B/05958/01 and by IAP 6-10 Photonics@be. K. De Vos and T. Claes thank the Flemish Institute for the Promotion of Innovation through Science and Technology (IWT) for a specialization grant.

\section{REFERENCES}

1. W. Bogaerts, R. Baets, P. Dumon, V. Wiaux, S. Beckx, D. Taillaert, B. Luyssaert, J. Van Campenhout, P. Bienstman, and D. Van Thourhout, "Nanophotonics waveguides in silicon-on-insulator fabricated with cmos technology," Journal of Lightwave Technology 23(1), pp. 401-412, 2005. 
2. S. Selvaraja, P. Jaenen, W. Bogaerts, D. Van Thourhout, and R. Baets, "Fabrication of photonic wire and crystal circuits in silicon-on-insulator using 193nm optical lithography," Journal of Lightwave Technology , accepted for publication.

3. F. Vollmer and S. Arnold, "Whispering-gallery-mode biosensing: label-free detection down to single molecules," Nature Methods 7(5), pp. 591-596, 2008.

4. A. Armani, R. Kulkarni, S. Fraser, R. Flagan, and K. Vahala, "Label-free, single-molecule detection with optical microcavities," Science 5839(317), pp. 783-787, 2007.

5. D.-X. Xu, A. Densmore, A. Delage, P. Waldron, R. McKinnon, S. Janz, J. Lapointe, G. Lopinski, T. Mischki, E. Pose, P. Cheben, and J. Schmid, "Folded cavity soi microring sensors for high sensitivity and real time measurement of biomolecular binding," Optics Express 9(16), pp. 15137-15148, 2008.

6. K. De Vos, J. Girones, S. Popelka, E. Schacht, R. Baets, and P. Bienstman, "Soi optical microring resonator with poly(ethylene glycol) polymer brush for label-free biosensor applications," Biosensors and Bioelectronics (24), p. 25282533, 2009.

7. K. De Vos, I. Bartolozzi, E. Schacht, P. Bienstman, and R. Baets, "Silicon-on-insulator microring resonator for sensitive and label-free biosensing," Optics Express 15, pp. 7610-7615, JUN 112007.

8. V. R. Almeida, Q. Xu, C. A. Barrios, and M. Lipson, "Guiding and confining light in void nanostructure," Optics Letters 29, pp. 1209-1211, june 2004.

9. C. A. Barrios, M. J. Banuls, V. Gonzalez-Pedro, K. B. Gylfason, B. Sanchez, A. Griol, A. Maquieira, H. Sohlstrom, M. Holgado, and R. Casquel, "Label-free optical biosensing with slot-waveguides," Optics Letters 33, pp. 708-710, APR 12008.

10. www.epixfab.eu.

11. H. Su and X. G. Huang, "Fresnel-reflection-based fiber sensor for on-line measurement of solute concentration in solutions," Sensors and Actuators B-Chemical 126, pp. 579-582, OCT 12007.

12. X. D. Hoa, A. G. Kirk, and M. Tabrizian, "Towards integrated and sensitive surface plasmon resonance biosensors: A review of recent progress," Biosensors \& Bioelectronics 23(2), pp. 151-160, 2007.

13. J. Homola, "Present and future of surface plasmon resonance biosensors," Anal. Bioanan. Chem. 377, pp. 528-539, 2003.

14. P. Debackere, S. Scheerlinck, P. Bienstman, and R. Baets, "Surface plasmon interferometer in silicon-oninsulator: novel concept for an integrated biosensor," Optics Express 14(16), pp. 7063-7072, 2006.

15. S. Selvaraja, E. Sleeckx, M. Schaekers, W. Bogaerts, D. Van Thourhout, P. Dumon, and R. Baets, "Low-loss amorphous silicon-on-insulator technology for photonics integrated circuitry," Optics Communications 182(9), pp. 1767-1770, 2009.

16. D. R. Lide, CRC Handbook of Chemistry and Physics, CRC Publishing Company, London, 2004.

17. C. Thirstrup and W. Zong, "Data analysis for surface plasmon resonance sensors using dynamic baseline algorithm," Sensors and Actuators B-Chemical 106(2), pp. 796-802, 2005. 